\title{
Comportement d'une dérivation de canaux lorsque les biefs aval sont pourvus de retenues
}

\author{
PAR
}

\author{
J. Nougaro \\ Professeur a l'Institut National Polytechnique de Toulouse \\ Directeur de l'rnstitut de Mecanique des Fluides de Toulouse \\ (Laboratoire associé au C.N.R.S.)

\section{P. Boyer ex J. Claria} \\ Ingénieurs au C.N.R.S.
}

\section{1 - Introduction}

Dans une étude antérieure $\left({ }^{1}\right)$, nous avons proposé une solution de première approximation pour résoudre le problème des dérivations de canaux découverts fonctionnant sans retenue dans les deux branches aval.

Afin d'étendre le domaine expérimental des régimes infracritiques dans les biefs constituant la dérivation, nous avons disposé à l'aval de chacun d'eux, une vanne mobile permettant de faire varier les tirants d'eau.

Dans cet article, nous fournissons des résultats qui définissent les paramètres hydrauliques caractérisant le fonctionnement d'une dérivation, à partir des conditions amont, et cela malgré l'introduction de deux variables supplémentaires que constituent les hauteurs de vannes.

Nous proposons également l'expression d'un coefficient

(1) J. Nougaro et P. Boyer : Sur la séparation des ealux dans les dérivations de canaux à section rectangulaire ( $L$ a Houille Blanche, n" $3 / 1974$ ).

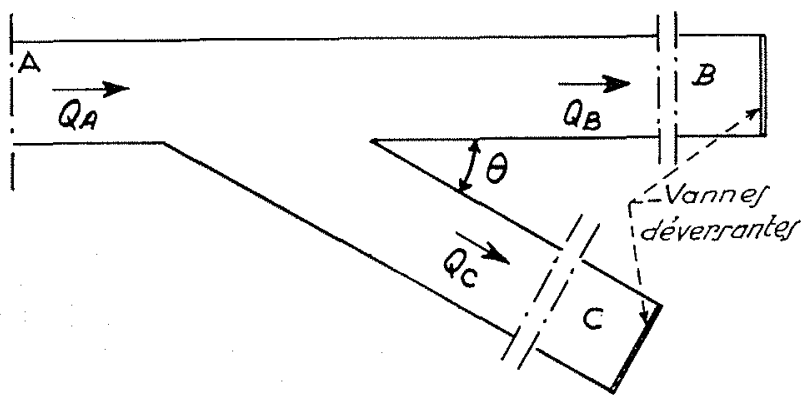

I/ mettant en évidence la perte d'énergie à la traversée de l'ouvrage.

Enfin, nous décrivons un phénomène d'instabilité qui prend naissance à l'intérieur de la branche dérivée, dans des conditions bien définies de fonctionnement.

\section{2 - Etude expérimentale - Construction d'un abaque}

C'est à partir de l'installation décrite dans l'article mentionné $\left({ }^{2}\right)$, que nous avons réalisé nos expériences. Rappelons que les canaux horizontaux de section rectangulaire forment trois biefs $A, B, C$, de même largeur et que la dérivation se situe sur la rive droite du canal principal (fig. 1). Dans cette étude, trois angles ont été étudiés: $\theta=30,45$ et $60^{\circ}$.

Les retenues sont matérialisées par des vannes déversantes, situées à l'extrémité aval des biefs secondaires. Ainsi, on est en présence d'une installation où les deux canaux $B$ et $C$ peuvent être assimilés à deux déversoirs dont les hauteurs de pelle, $P_{B}$ et $P_{C}$, changent pour chaque condition de réglage, ce qui conduit, compte tenu des variations possibles de l'énergie amont, à une infinité de solutions.

Nous avons étudié, en tout premier lieu, l'aspect des écoulements dans les divers éléments du dispositif expérimental et ceci nous a permis de constater :

- que le régime supracritique n'est jamais atteint dans aucun des biefs;

(2) Loc. cit. 


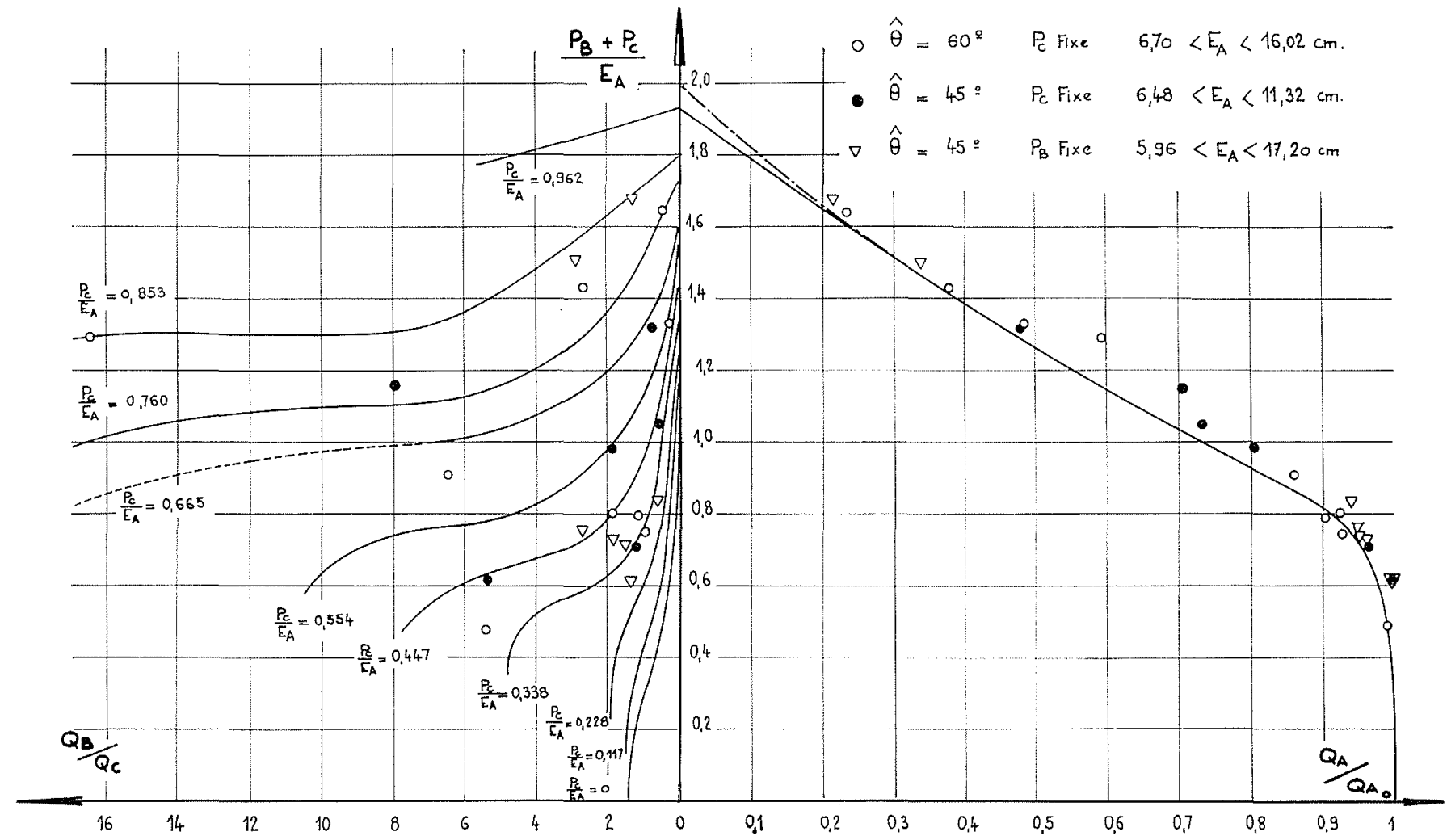

2/

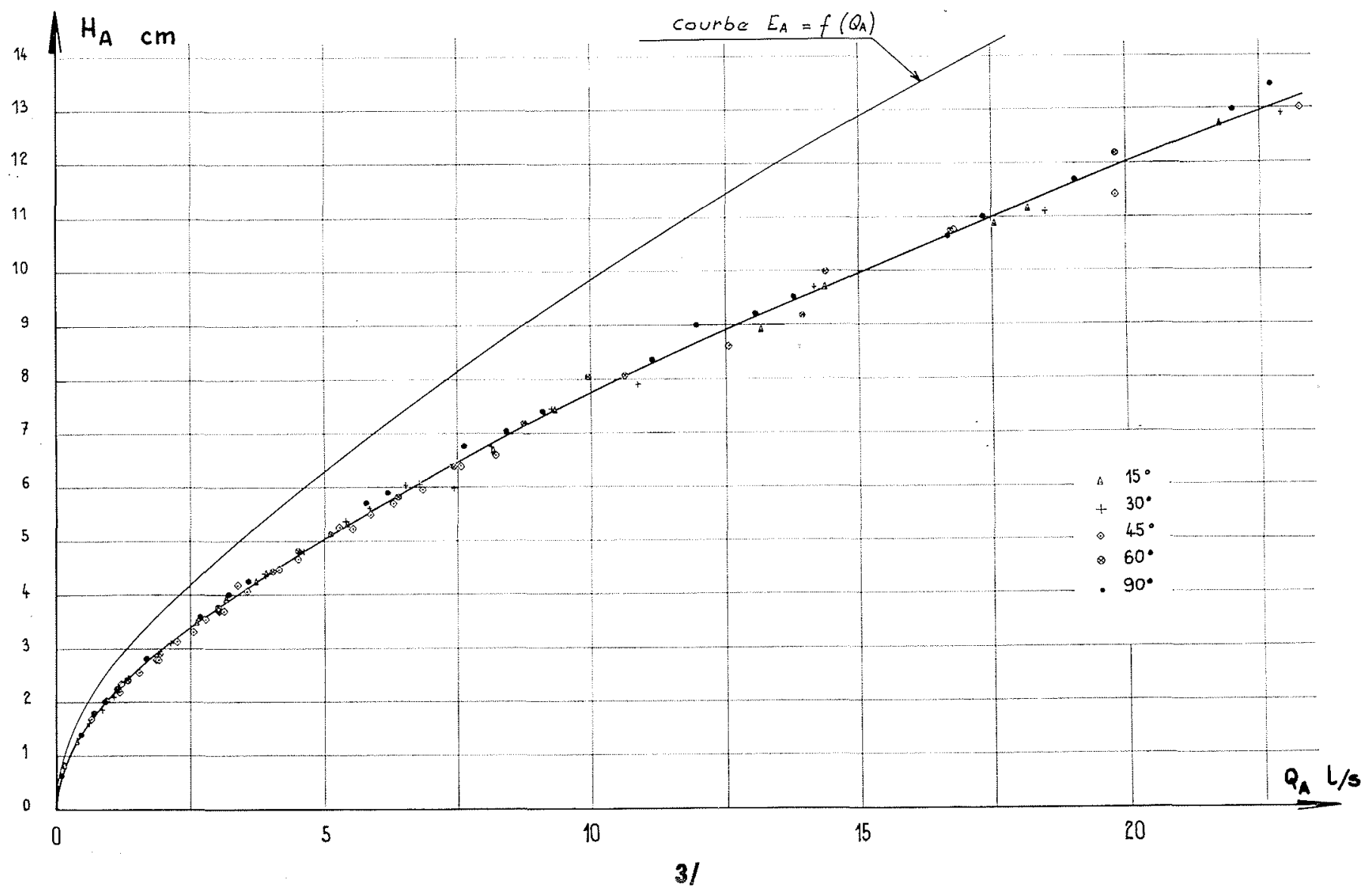


- que l'angle de dérivation n'a aucune influence sur la loi de fonctionnement amont.

Par suite, dans les biefs aval $B$ et $C$, on remarque qu'il est possible de remplacer la loi $Q=f(H)$ par $Q=f(E-P)$, cette dernière étant la loi de fonctionnement des déversoirs.

Nous avons alors vérifié que pour tout angle $\theta$ de dérivation et pour toute valeur des hauteurs de retenues, le nombre de Froude dans les biefs $B$ et $C$ pouvait s'exprimer par :

$$
F_{B}=F_{C}=0,772 \log E / P
$$

$E$ étant l'énergie spécifique dans le canal considéré, $P$ la hauteur de vanne.

Il faut tout de même remarquer que, pour une condition de fonctionnement quelconque, $F_{B}$ sera toujours différent de $F_{C}$ puisque $P_{B}$ est différent de $P_{C}$ et, par voie de conséquence, $E_{B}$ différent de $E_{C}$.

A partir d'une série d'expériences effectuées avec une dérivation d'angle $\theta=30^{\circ}$, cent combinaisons ont été obtenues par la modification successive de $P_{B}$ puis de $P_{C}$ en conservant à $E_{A}$ une valeur constante, $\left(P_{B}\right.$ et $P_{C}$ variant dans les limites 0 à $E_{A}$ ). Ceci nous a permis de tracer deux représentations graphiques définies par:

$$
\left(P_{B}+P_{C}\right) / E_{A}=\Phi_{1}\left(Q_{A} / Q_{A_{0}}\right)
$$

où $Q_{A_{0}}$ représente le débit que l'on obtiendrait dans le canal principal si les biefs secondaires étaient dépourvus de retenues et

$$
\left(P_{B}+P_{C}\right) / E_{A}=\Phi_{2}\left(Q_{B} / Q_{C}\right)
$$

où $Q_{B} / Q_{C}$ est le rapport de partage des débits secondaires.

Les équations (2) et (3) ayant le premier membre commun, on peut envisager de les jumeler avec la même ordonnée $\left(P_{B}+P_{C}\right) / E_{A}$.

La figure 2 présente cette forme d'abaque qui comme nous le verrons peut être généralisée à tous les cas.

Sur la partie droite de l'abaque représentant la fonction (2) on remarque que le rapport $\left(P_{B}+P_{C}\right) / E_{A}$ atteint la valeur 2 lorsque les hauteurs de pelles sont chacune égale à la valeur qui mesure l'énergie amont $E_{A}$ et dans ces conditions il n'y a plus d'écoulement on a $Q_{A} / Q_{A_{0}}=0$.

Par contre, lorsque les vannes sont totalement effacées $P_{I B}=P_{C}=0, \quad Q_{A} / Q_{A_{0}}=1$.

Sur la deuxième partie du graphique où est portée la fonction (3) on a obtenu un faisceau de courbes caractérisées par $P_{C} / E_{A}=$ Cte. Le lieu des débits maximum dans le bief $B$ est donné par la courbe $P_{B}=0$, qui aboutit avec la courbe $P_{C}=0$ au point d'abscisse $Q_{B} / Q_{C}=1,375$. C'est la condition que nous avons obtenue pour le partage des débits lorsqu'il n'y a pas de retenues aval $\left({ }^{3}\right)$.

\begin{tabular}{|c|c|c|c|c|c|c|c|c|c|}
\hline$\hat{\theta}$ & $\begin{array}{c}E_{A} \\
(\mathrm{~cm})\end{array}$ & $Q_{A} / Q_{A_{0}}$ & $\begin{array}{c}P_{B} \\
(\mathrm{~cm})\end{array}$ & $\begin{array}{c}P_{C} \\
(\mathrm{~cm})\end{array}$ & $\frac{P_{B}+P_{C}}{E_{A}}$ & $Q_{B} / Q_{c}$ & $\underset{\text { vrai }}{P_{C} / E_{A}}$ & $\begin{array}{c}P_{C} / E_{A} \\
\text { interpolé }\end{array}$ & $\begin{array}{c}\text { ECARTS } \\
(\%)\end{array}$ \\
\hline \multirow{8}{*}{$60^{\circ}$} & 6,70 & 0,234 & 6 & 5 & 1,642 & 0,421 & 0,747 & 0,760 & $+1,75$ \\
\hline & 13,84 & 0,904 & 6 & 5 & 0,794 & 1,125 & 0,361 & 0,369 & $+2,2$ \\
\hline & 7,72 & 0,866 & 2 & 5 & 0,907 & 6,48 & 0,648 & 0,620 & $-4,3$ \\
\hline & 14,54 & 0,988 & 2 & 5 & 0,482 & 5,39 & 0,344 & 0,386 & +12 \\
\hline & 11,26 & 0,925 & 4 & 5 & 0,800 & 1,97 & 0,447 & 0,445 & $-0,45$ \\
\hline & 16,05 & 0,927 & 7 & 5 & 0,748 & 0,958 & 0,312 & 0,323 & $+3,5$ \\
\hline & 9,77 & 0,483 & 8 & 5 & 1,330 & 0,264 & 0,512 & 0,535 & $+4,5$ \\
\hline & 12,60 & 0,381 & 8 & 10 & 1,430 & 2,657 & 0,794 & 0,789 & $-0,63$ \\
\hline \multirow{6}{*}{$45^{\circ}$} & 11,38 & 1,01 & 2 & 5 & 0,615 & 5,34 & 0,439 & 0,440 & $+0,23$ \\
\hline & 9,17 & 0,806 & 4 & 5 & 0,982 & 1,923 & 0,545 & 0,555 & $+1,84$ \\
\hline & 8,32 & 0,481 & 6 & 5 & 1,318 & 0,700 & 0,602 & 0,626 & $+4,0$ \\
\hline & 15,66 & 0,966 & 6 & 5 & 0,703 & 1,18 & 0,319 & 0,333 & $+4,4$ \\
\hline & 12,36 & 0,736 & 8 & 5 & 1,051 & 0,516 & 0,404 & 0,415 & $+2,73$ \\
\hline & 6,48 & 0,708 & 2,5 & 5 & 1,158 & 7,97 & 0,772 & 0,770 & $-0,35$ \\
\hline \multirow{7}{*}{$45^{\circ}$} & 1,00 & 0,216 & 5 & 5 & 1,678 & 1,270 & 0,838 & 0,847 & $+1,07$ \\
\hline & 16,62 & 0,968 & 5 & y & 0,712 & 1,408 & 0,356 & 0,359 & $+0,84$ \\
\hline & 7,40 & 0,940 & 5 & 2 & 0,834 & 0,510 & 0,238 & 0,257 & $+8,0$ \\
\hline & 19,05 & 1,004 & 5 & 4 & 0,603 & 1,323 & 0,267 & 0,276 & $+3,27$ \\
\hline & 2,18 & 0,341 & 5 & 6 & 1,505 & 2,868 & 0,820 & 0,821 & $+0,12$ \\
\hline & 18,10 & 0,955 & 5 & 6 & 0,734 & 1,784 & 0.401 & 0,410 & $+2,24$ \\
\hline & 22,35 & 0,950 & 5 & 8 & 0,756 & 2,604 & 0,465 & 0,463 & $-0,43$ \\
\hline
\end{tabular}

(3) Loc. cit.

\section{Tableau 1}



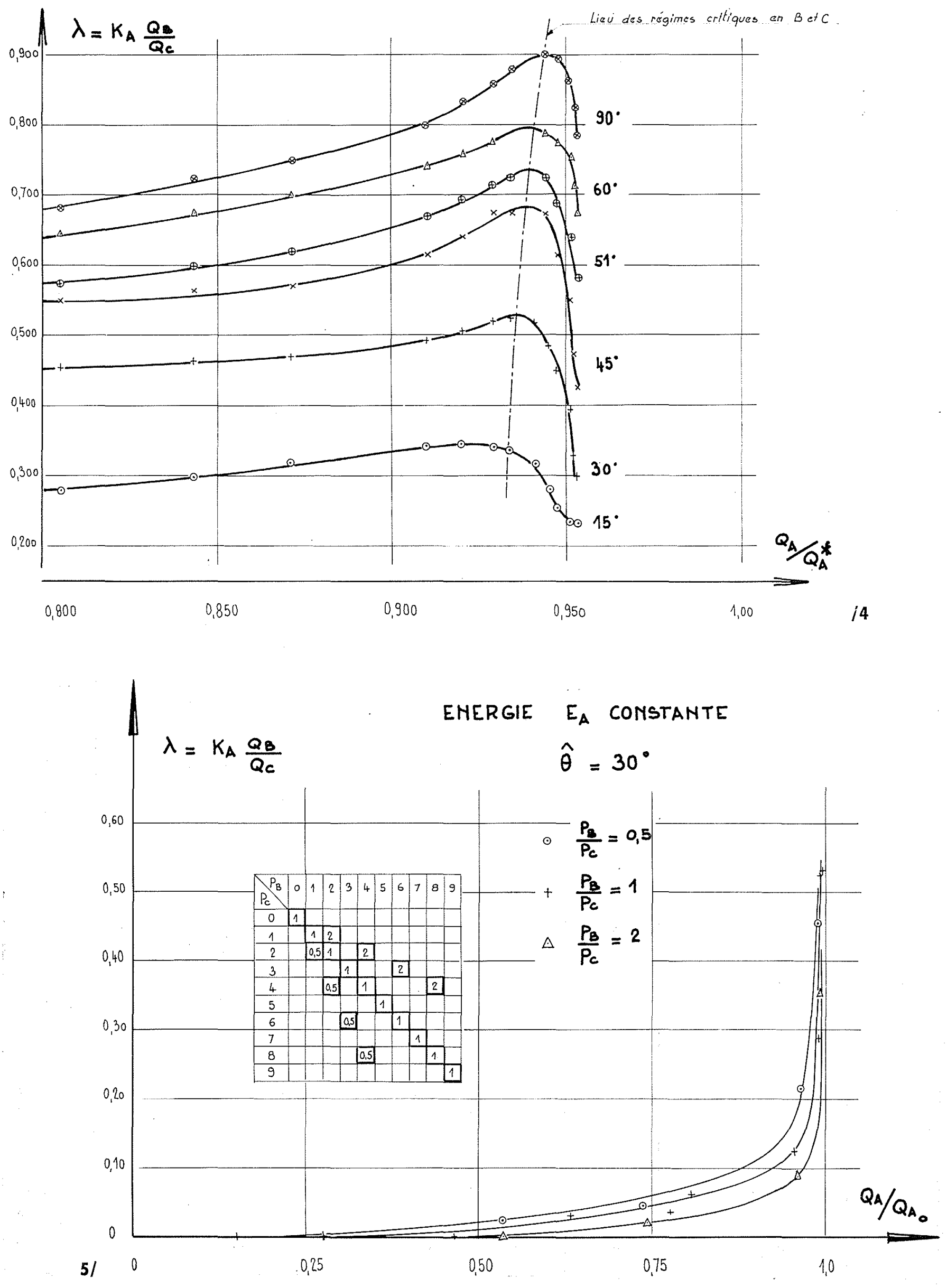
Connaissant les conditions amont $Q_{A}$ et $H_{A}$ et l'une des valeurs aval, hauteur de pelle ou débit dérivé, il est facile de déduire les autres éléments du fonctionnement de l'ouvrage en utilisant judicieusement l'abaque.

Les conditions amont permettent de trouver le débit de référence $Q_{A_{0}}$ qui s'écoulerait dans le canal, lorsque les biefs $B$ et $C$ n'ont pas de retenue. Il est alors facile de déterminer l'énergie $E_{A_{0}}$ et, par suite, le débit $Q_{A_{0}}$ à partir de la courbe $E_{A_{0}}=f\left(Q_{.1}\right)$ (fig. 3).

\section{$1^{\text {er }}$ PROBLÈME :}

Le troisième paramètre connu est la levée de vanne.

On part du point 1 d'abscisse $Q_{A} / Q_{A_{0}}$ à partir duquel on déduit $\left(P_{R}+P_{C}\right) / E_{A}$. Comme $P_{C} / E_{A}$ est connu on calcule $P_{B}$ et en utilisant l'abaque on trouve $Q_{B} / Q_{C}$ donc $Q_{B}$ et $Q_{C}$.

Si c'est $P_{l}$ qui est donné, on procède de la même façon puisque $Q_{A} / Q_{A_{0}}$ permet de trouver $\left(P_{B}+P_{C}\right) / E_{A}$ donc $P_{C} / E_{\text {: }}$.

\section{$2^{\circ}$ PROBLÈME:}

La troisième donnée est le débit $Q_{B}$ ou $Q_{C}$.

On calcule $Q_{B} / Q_{O}$ et par suite à partir de 2 on détermine $\left(P_{I B}+P_{C}\right) / E_{A 1}$ d'une part et $P_{C} / E_{A}$ d'autre part.

Nous avons voulu vérifier la validité de cet abaque pour des conditions expérimentales totalement différentes.

Pour cela nous avons fixé deux. valeurs de l'angle de dérivation soit $\theta=45$ et $60^{\circ}$ et nous avons fait varier l'énergie amont $E_{A}$.

La comparaison de $P_{C} / E_{A}$ expérimental avec $P_{C} / E_{A}$ défini à partir de notre abaque montre, par le tableau 1 , que la moyenne des écarts obtenus est sensiblement égale à $5 \%$.

\section{3 - Dissipation de l'énergie dans une dérivation}

Lorsqu'une singularité est traversée par un courant, il se produit une dissipation d'énergie qui dépend de la géométrie de l'ouvrage.

Dans une dérivation, il ne paraît pas logique de nommer cette dissipation «perte de charge ». En effet, il n'est pas possible de comparer l'énergie spécifique amont à la somme des énergies spécifiques dans les branches dérivées. Par contre, on pourra montrer l'efficacité de l'ouvrage en affectant aux énergies issues de chaque bief un coefficient $\xi$.

Lorsque les biefs sont dépourvus de retenues, les énergies totales en poids dans leur section droite respective s'écrivent :

$$
\begin{aligned}
& \rho g Q_{A} E_{A}=\rho g Q_{A}\left(H_{A}+\alpha_{A} V_{A}^{2 / 2} g\right) \\
& \rho g Q_{B} E_{B}=\rho g Q_{B}\left(H_{B}+\alpha_{B} V_{B}^{2 / 2} g\right) \\
& \rho g Q_{C} F_{C}=\rho g Q_{C}\left(H_{C}+\alpha_{C} V_{C}^{2 / 2} g\right)
\end{aligned}
$$

Le principe de conservation de l'énergie permet d'écrire dans le cas où on fait ressortir la perte de charge dans $B$ et dans $C$ :

$$
\begin{aligned}
Q_{A}\left(H_{A}+\alpha_{A} V_{A}^{2} / 2 \mathrm{~g}\right)= & Q_{B}\left(H_{B}+\alpha_{B} V_{B^{2}} / 2 \mathrm{~g}+\xi_{B}\right) \\
& +Q_{C}\left(H_{C}+\alpha_{C} V_{C^{2}} / 2 g+\xi_{C}\right) \\
&
\end{aligned}
$$

En supposant que le mouvement s'effectue en bloc dans les trois biefs on a $\alpha_{\mathrm{L}}=\alpha_{B}=\alpha_{C}=1$

et $Q_{A}\left(H_{A}+V_{A}^{2} / 2 g\right)-Q_{B}\left(H_{B}+V_{B}^{2} / 2 g\right)$

$$
+Q_{C}\left(H_{C}+V_{C}^{2} / 2 \mathrm{~g}\right)=Q_{B} \xi_{B}+Q_{C} \xi_{C}
$$

Le deuxième membre de cette expression représente la perte d'énergie totale à la traversée de la dérivation.

On peut alors définir un coefficient d'efficacité $K_{A}$ de la singularité en ramenant cette perte totale à l'énergie amont, on a alors :

$$
K_{A}=\frac{Q_{A} E_{A}-\left(Q_{B} E_{B}+Q_{C} E_{C}\right)}{Q_{A} E_{A}}=\frac{Q_{B} \xi_{B}+Q_{C} \xi_{C}}{Q_{A} E_{A}}
$$

En soulignant que le terme $\left(Q_{B} E_{B}+Q_{C} E_{C}\right) / Q_{A} E_{A}$ peut représenter le rendement $\eta$ de la singularité on peut écrire :

$$
\eta=1--K_{A}
$$

Nous avons pu constater qu'il existait un coefficient d'efficacité pour chaque angle $\theta$ et qu'il évoluait avec le débit $Q_{A}$.

Pour rendre ces résultats exploitables, nous avons voulu proposer une représentation en valeurs adimensionnelles.

Puisque $K_{A}$ dépend de l'angle $\theta$ tout comme le rapport de partage des débits, nous avons pensé que finalement $Q_{B} / Q_{C}$ pouvait représenter un coefficient de forme puisqu'il caractérise la géométrie de la prise.

On a ainsi obtenu un coefficient de dissipation tel que :

$$
\lambda=K_{A} \times\left(Q_{B} / Q_{C}\right)
$$

La représentation de ce paramètre en fonction de $Q_{A} / Q_{A}{ }^{*}$ est donnée par la figure 4.

A partir de l'instant où l'on obstrue les biefs par des vannes aux extrémités de $B$ et $C$, on repose le problème sur le plan énergétique.

Les débits secondaires peuvent alors s'exprimer par :

$$
Q=m \cdot B \cdot \sqrt{2} g \cdot(E-P)^{3 / 2}
$$

En première approximation, $m \cdot B \cdot \sqrt{2} g=k$ et les énergies dans les biefs $B$ et $C$ s'écrivent:

et :

$$
E_{B}=\left(Q_{B} / k\right)^{2 / 3}+P_{B}
$$

$$
E_{O}=\left(Q_{c} / k\right)^{2 / 3}+P_{C}
$$

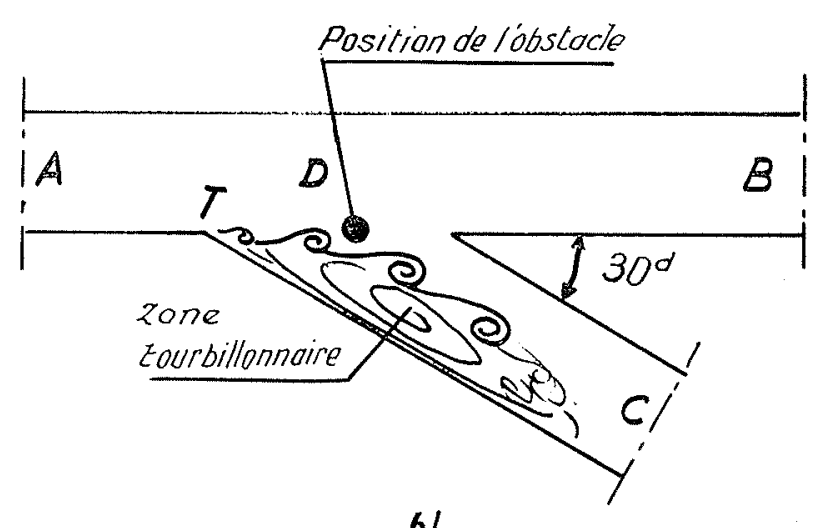

6) 


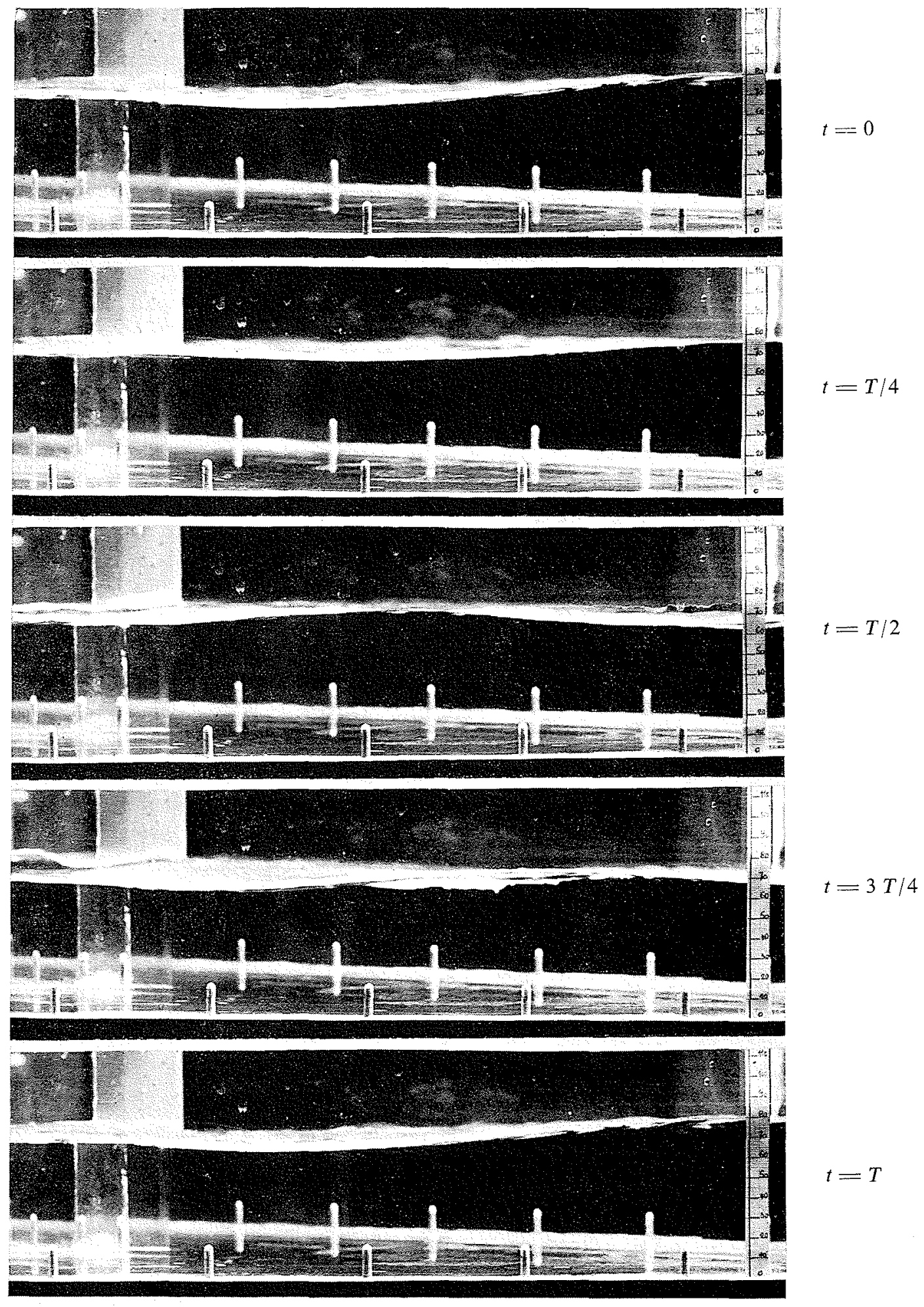


dans ce cas le coefficient d'efficacité s'écrit:

$$
K_{A}=1-\frac{Q_{B}\left[\left(Q_{B} / k\right)^{2 / 3}+P_{B}\right]-Q_{C}\left[\left(Q_{C} / k\right)^{2 / 3}+P_{C}\right]}{\left(Q_{B}+Q_{C}\right) E_{A}}
$$

Comme précédemment, il peut être affecté du coefficient de forme $Q_{B} / Q_{C}$ et l'on retrouve le coefficient de dissipation $\lambda=K_{A} \times\left(Q_{B} / Q_{C}\right)$.

La représentation de $\lambda$ en fonction de $Q_{A} / Q_{A_{0}}$ n'a été possible que dans les limites du rapport $P_{B} / P_{C}$ :

$$
0,5<P_{B} / P_{C}<2
$$

En effet, au-delà de ces valeurs, il se produit un phénomène d'instabilité que nous décrirons plus loin.

La figure 5 montre l'évolution de $\lambda$ en fonction de $Q_{A} / Q_{A_{0}}$.

Lorsque $Q_{A} / Q_{A_{0}}$ est inférieur à 0,95 , le coefficient est relativement faible, ce qui souligne l'influence prépondérante des retenues. Au-delà de $Q_{A} / Q_{A_{0}}=0,95, \lambda$ croît très rapidement pour atteindre la valeur limite fixée par $P_{B}=P_{C}=0$ soit $Q_{A}=Q_{A_{0}}$.

\section{PHÉNOMẼNE OSCILLATOIRE}

Nous avons vu qu'au cours de notre étude expérimentale, nous avions été amenés à considérer une succession de hauteurs de vannes installées sur les deux biefs aval.

Dans le cas où, sur le canal $B$, la vanne était complètement effacée, nous avons remarqué qu'à partir d'une certaine valeur du tirant d'eau dans la branche $C$ un phénomène d'oscillation longitudinale prenait naissance dans cette partie de l'ouvrage et se propageait jusqu'au droit de la dérivation.

L'examen de l'écoulement, visualisé à l'aide d'un colorant, nous a permis d'établir la cause engendrant ce phénomène.

A l'amont immédiat de la branche dérivée, il se produit un décollement et une zone tourbillonnaire prend naissance, séparée de la veine correspondant au mouvement direct par une surface de discontinuité de la vitesse.

Nous avons constaté que cette surface de discontinuité, au lieu de conserver un aspect régulier et stable, était perturbée par des tourbillons qui se forment périodiquement au point $T$, rencontre du courant de retour appartenant à la zone tourbillonnaire et du courant provenant du canal principal $A$ (fig. 6).

Ces tourbillons, qui à l'origine sont peu importants, se développent au fur et à mesure qu'ils progressent à l'intérieur du canal dérivé, provoquant, lors de leur passage au droit de la section contractée, une modification de la largeur de celle-ci et par suite du débit admis dans la branche $C$.

Cette fluctuation du débit semble être à l'origine des oscillations que nous avons observées.

En effet, si, au moyen d'obstacles convenablement placés, on contrarie le développement des tourbillons secondaires qui se greffent sur le contour de la zone de décollement, on s'aperçoit que le débit dans la branche $C$ reprend une valeur relativement constante et que les oscillations du plan d'eau disparaissent. Ce résultat a été obtenu, en particulier, en plaçant au droit de la prise, comme il est indiqué sur la figure 6 , un obstacle cylindrique de $4 \mathrm{~cm}$ de diamètre.

Nous avons remarqué que, pour une hauteur de déversoir $P_{C}$ sensiblement égale à $2 E_{A} / 3$, le phénomène d'oscillation atteignait son amplitude maximale. Dans ce cas, nous sommes arrivés à développer des fluctuations assez fortes pour que le plan d'eau, dans son mouvement descendant, arrive en contrebas du seuil, ce qui provoque par intermittences l'arrêt du débit déversé.

La série de photographies de la figure 7 montre l'évolution du plan d'eau au droit de la prise durant une période d'oscillation.

Dans le cas présent nous avions :

- énergie $E_{A}$ dans la branche amont $\ldots \ldots 9,43 \mathrm{~cm}$

- hauteur du déversoir $P_{C} \ldots \ldots \ldots \ldots, 00 \mathrm{~cm}$

- différence entre les valeurs extrêmes du plan d'eau au cours d'une oscillation ... 2,50 cm

— fréquence des oscillations .......... 0,4 Hertz

En opérant avec différentes valeurs de l'énergie amont $E_{A}$ nous avons vu que, d'une part, on pouvait obtenir des oscillations dont l'importance augmentait au fur et à mesure que $E_{A}$ devenait plus grand et que, d'autre part, la fréquence du phénomène conservait sensiblement la même valeur, dans les limites de nos expériences, c'est-à-dire jusqu'à une valeur de $E_{A}$ égale à $15 \mathrm{~cm}$. 